Research article Open Access

\title{
MUC1 alters oncogenic events and transcription in human breast cancer cells
}

\author{
Christine L Hattrup and Sandra J Gendler
}

Mayo Clinic College of Medicine, Mayo Clinic Arizona, Scottsdale, AZ 85259, USA

Corresponding author: Sandra J Gendler, gendler.sandra@mayo.edu

Received: 9 Apr 2006 Revisions requested: 3 May 2006 Revisions received: 14 Jun 2006 Accepted: 20 Jun 2006 Published: 17 Jul 2006

Breast Cancer Research 2006, 8:R37 (doi:10.1186/bcr1515)

This article is online at: http://breast-cancer-research.com/content/8/4/R37

(c) 2006 Hattrup and Gendler; licensee BioMed Central Ltd.

This is an open access article distributed under the terms of the Creative Commons Attribution License (http://creativecommons.org/licenses/by/2.0), which permits unrestricted use, distribution, and reproduction in any medium, provided the original work is properly cited.

\begin{abstract}
Introduction MUC1 is an oncoprotein whose overexpression correlates with aggressiveness of tumors and poor survival of cancer patients. Many of the oncogenic effects of MUC1 are believed to occur through interaction of its cytoplasmic tail with signaling molecules. As expected for a protein with oncogenic functions, MUC1 is linked to regulation of proliferation, apoptosis, invasion, and transcription.
\end{abstract}

Methods To clarify the role of MUC1 in cancer, we transfected two breast cancer cell lines (MDA-MB-468 and BT-20) with small interfering (si)RNA directed against MUC1 and analyzed transcriptional responses and oncogenic events (proliferation, apoptosis and invasion).

Results Transcription of several genes was altered after transfection of MUC1 siRNA, including decreased MAP2K1 (MEK1), JUN, PDGFA, CDC25A, VEGF and ITGAV (integrin $\alpha_{v}$ ), and increased $T N F, R A F 1$, and MMP2. Additional changes were seen at the protein level, such as increased expression of c-Myc, heightened phosphorylation of AKT, and decreased activation of MEK $1 / 2$ and ERK1/2. These were correlated with cellular events, as MUC1 siRNA in the MDA-MB-468 line decreased proliferation and invasion, and increased stressinduced apoptosis. Intriguingly, BT-20 cells displayed similar levels of apoptosis regardless of siRNA, and actually increased proliferation after MUC1 siRNA.

Conclusion These results further the growing knowledge of the role of MUC1 in transcription, and suggest that the regulation of MUC1 in breast cancer may be more complex than previously appreciated. The differences between these two cell lines emphasize the importance of understanding the context of cellspecific signaling events when analyzing the oncogenic functions of MUC1, and caution against generalizing the results of individual cell lines without adequate confirmation in intact biological systems.

\section{Introduction}

MUC1 is the founding member of the mucin family: proteins characterized by heavy O-glycosylation centering around a variable number of tandem repeats that are rich in serine and threonine residues [1,2]. MUC1 is a transmembrane heterodimer with one subunit solely extracellular (MUC1-EX), and the other subunit composed of a short extracellular stem, a single transmembrane domain, and the cytoplasmic tail (together called the MUC1-CT). MUC1 possesses both pro- and antiadhesive capacities, as the MUC1-EX provides binding sites for a variety of adhesion proteins, while its large size and extended structure prevents cell-cell contact [3-5].
Initially described as a tumor antigen overexpressed in $>90 \%$ of breast cancers, MUC1 is now known to be an oncogene with roles in both tumor formation and progression $[1,6]$. Mouse studies have been integral to the current understanding of MUC1 in cancer. Muc1 knockout mice (Muc1 ${ }^{-/} ;$MUC1 is human; Muc1 is mouse) show a reduction in tumorigenic phenotype when crossed onto mice overexpressing the Wnt1 [7] or polyomavirus middle T antigen [8] oncogenes in the mammary gland. In contrast, MUC1 overexpression in the mammary gland drives tumor formation [9], indicating that MUC1 is a true oncogene.

Many of the oncogenic effects of MUC1 stem from its cytoplasmic tail, which binds to several proteins implicated in can-

$\mathrm{BrdU}=$ bromodeoxyuridine; $\mathrm{EGF}=$ epidermal growth factor; EGFR = epidermal growth factor receptor; ERK = extracellular signal regulated kinase; MAPK = mitogen activated protein kinase; MEK = MAPK and ERK kinase; MMP = matrix metalloproteinases; MUC1-CT = MUC1 cytoplasmic tail; MUC1-EX = MUC1 extracellular subunit; PBS = phosphate-buffered saline; PI = propidium iodide; siRNA = small interfering RNA; TIMP = tissue inhibitor of metalloproteinases; TNF = tumor necrosis factor. 
cer, including c-Src $[10,11]$ and the epidermal growth factor receptor (EGFR) family $[12,13]$. MUC1 stimulates mitogen activated protein kinase (MAPK) signaling through the extracellular signal regulated kinases (ERK1/2) [12,14]; this can occur through MUC1 association with Grb2 and son of sevenless to activate Ras [15]. ERK $1 / 2$ signaling is commonly stimulated by the Ras-Raf-MEK (MAPK and ERK kinase) cascade downstream of mitogens such as EGFR [16], and regulates transcription via factors like the activator protein-1 complex. Loss of MUC1 can reduce EGFR expression [17], providing another means of affecting MAPK signaling. Our results describe a novel mechanism by which MUC1 regulates the ERK1/2 pathway, through modulating transcription of the genes encoding MEK1, Raf-1, and c-Jun.

MUC1 expression correlates with increased survival in response to cytotoxic or oxidative agents [18-21], and can activate the phosphoinositol-3 kinase-AKT pathway as part of an anti-apoptotic response [18]. MUC1 has also recently been linked to transcription, as the MUC1-CT localizes to the nucleus [22] and affects transcription by $\beta$-catenin $[22,23]$, FOXO3a [21], p53 [24], and estrogen receptor $\alpha$ [25]. However, there are indications that the role of MUC1 in oncogenesis is regulated by cell type and signaling context. For example, MUC1 can stimulate Fas-mediated apoptosis [26], while Muc1 is specifically down-regulated in c-neu-induced mammary tumors [27]. This report emphasizes the complexity of MUC1 signaling in breast cancer by contrasting results from two established breast cancer cell lines.

To understand MUC1 function in cells with high endogenous expression, that is, cells likely to have evolved with active MUC1 signaling, we used small interfering RNA (siRNA) to knock down MUC1 in MDA-MB-468 and BT-20 cells. We then analyzed transcription of 84 genes involved in cancer, as well as the effects upon cellular events linked to oncogenesis, such as apoptosis and proliferation. Though the cell lines show some similarity in transcriptional alterations after transfection with MUC1 siRNA, their phenotypes are quite dissimilar: MDA-MB-468 increases apoptosis and reduces proliferation and invasion, while BT-20 proliferates more rapidly after loss of MUC1. This last may reflect the striking amount of active AKT in BT-20; AKT activity is increased in both cell lines after MUC1 siRNA, which agrees with a previous study of MUC1 siRNA [21], but disagrees with results from 3Y1 fibroblasts [18]. Recent studies have emphasized the complex and context-specific regulation of even such classic oncogenes as AKT [28]. The differences between the two breast cancer cell lines in this study suggest that MUC1 oncogenic functions are also subject to cell-specific regulation, and stress the need for understanding the cellular signaling context when interpreting results.

\section{Materials and methods Cell culture and siRNA transfection}

MDA-MB-468 and BT-20 cells (American Type Culture Collection) were cultured in Dulbecco's modified Eagle's medium (Invitrogen, Carlsbad, CA, USA) plus 10\% fetal calf serum, 1\% Glutamax (Invitrogen) and 1\% penicillin/streptomycin. Stable cell lines (468.Neo and 468.MUC1 $\Delta 8$ ) were selected with 0.5 $\mathrm{mg} / \mathrm{ml} \mathrm{G} 418$. For epidermal growth factor (EGF) stimulation, MDA-MB-468 cells were serum-starved overnight and treated for 10 minutes at $37^{\circ} \mathrm{C}$ with $100 \mathrm{ng} / \mathrm{ml}$ EGF. Transient siRNA transfection was performed with Lipofectamine2000 (Invitrogen) and $100 \mathrm{nM}$ siRNA oligonucleotides. The commercially available siRNA constructs (all from Dharmacon, Lafayette, CO, USA) were scrambled (siCONTROL non-targeting siRNA \#1), or directed against firefly luciferase (siCONTROL non-targeting siRNA \#2) or MUC1 (siGENOME smartpool). The independent oligonucleotides designed in our laboratory target sequences beginning at MUC1 codons 882 and 956, and have been described previously [29]. The scrambled siRNA construct was used only in BT-20 cells as it causes a non-specific knockdown of MUC1 in the MDA-MB-468 line.

\section{Cloning of MUC1 WT vector and stable transfection}

Two silent mutations (G891A and T894C) were introduced into the MUC1 cDNA (called MUC1 $\Delta 8$ ) to make it resistant to the 882 siRNA that targets that region of the mRNA. The mutant cDNA was cloned into the pLNCX.1 vector with neomycin resistance (gift of Joseph Loftus, Mayo Clinic, Arizona, USA). Stable transfection was performed with Lipofectamine2000; cells were selected beginning 24 hours post-transfection and maintained as a polyclonal population.

\section{Western blots and antibodies}

Cells were lysed in buffer (20 mM HEPES pH 8.0, $150 \mathrm{mM}$ sodium chloride, $1 \%$ Triton X-100, 2 mM EDTA) with commercial protease (Complete inhibitor cocktail, Roche, Pleasanton, CA, USA) and phosphatase inhibitors ( $10 \mathrm{mM}$ sodium fluoride, $2 \mathrm{mM}$ sodium vanadate, $50 \mu \mathrm{M}$ ammonium molybdate). Protein concentration was determined by BCA (Pierce, Rockford, IL, USA); $50 \mu \mathrm{g}$ of lysate were loaded on SDS-PAGE gels for each experiment, except for the pMEK1/2 blot in MDA-MB468 , where $150 \mu \mathrm{g}$ were used. Non-commercial antibodies used were: BC2, a mouse monoclonal to the MUC1-EX (gift of Dr McGuckin, Queensland University, Queensland, Australia), and CT2, an Armenian hamster monoclonal to the MUC1-CT developed in our lab [12]. Antibodies to pMEK1/2, MEK1/2, ERK1/2, Myc, pAKT, AKT, $\beta$-tubulin (all Cell Signaling, Danvers, MA, USA), $\beta$-actin and dpERK $1 / 2$ (both Sigma, St. Louis, MO, USA) were used according to manufacturers' recommendations. All antibodies except $\beta$-actin $(1: 2,500)$ and dpERK1/2 $(1: 10,000)$ were used at 1:1,000 dilution for western blots. Flow cytometric analysis of MUC1 was done with HMPV-FITC, which recognizes the core peptide of the MUC1EX tandem repeats (Pharmingen, San Diego, CA, USA). Bromodeoxyuridine (BrdU) staining was performed with a fluores- 
cently conjugated antibody to BrdU (BrdU-PE, BD Biosciences, San Diego, CA, USA) as described below. Densitometry was performed using the public domain ImageJ program (developed at the $\mathrm{NIH}$ and available at [30]. Each band was measured in three places; the results were averaged and normalized to tubulin to control for loading.

\section{Transwell invasion assays}

Cells were serum-starved beginning 24 hours post-siRNA transfection. Cells were re-plated in serum-free medium 48 hours post-transfection at 50,000 cells per insert (sized for 24-well plates), with serum-containing medium in the bottom of the growth well as an attractant. Transwell inserts (BD Biosciences) pre-coated with laminin, fibronectin, collagen IV, or control (no matrix) were used, and cells were permitted to invade for 48 hours. At this point (96 hours post-transfection), visual inspection of the growth wells confirmed that negligible numbers of cells went through to the bottom of the plate. Noninvaded cells were swabbed from the tops of half of the inserts ('samples', containing only invaded cells), and retained in the others ('controls', all cells). Inserts were stained for 10 minutes with crystal violet $(0.5 \%$ in $20 \%$ methanol) and washed with water. Membranes were cut out and destained for 10 minutes in $10 \%$ acetic acid in a 96-well plate; membranes were removed and absorbance was read at $570 \mathrm{~nm}$. Percent invasion is defined as (absorbance of samples/absorbance of controls) $\times 100$.

\section{[3 ${ }^{3}$ ]Thymidine incorporation assays}

Cells were re-plated in quadruplicate 24 hours post-siRNA transfection at 15,000 cells/well (96-well plate) with $\left[{ }^{3} \mathrm{H}\right]$ thymidine $(1 \mu \mathrm{Ci} /$ well), then incubated in normal conditions for 24 hours. At this time (48 hours post-siRNA transfection) excess radioactivity was washed off and the cells were harvested and read on a TopCount plate reader. Statistical analysis was performed using JMP 5.1.2 software (SAS Institute, Inc., Cary, $\mathrm{NC}$, USA); the student's $t$ test was used to determine $p$ values and significance was confirmed with Wilcoxon rank sum and Pearson chi squared analyses.

\section{BrdU incorporation}

BrdU $(50 \mu \mathrm{M})$ was given to cells 48 hours post-siRNA transfection and permitted to incorporate for 1.5 hours. Cells were then washed with PBS, trypsinized, and washed again. BrdU staining was performed according to an adaptation of the manufacturer's protocol: cells were re-suspended in PBS, mixed $1: 1$ with $-20^{\circ} \mathrm{C}$ neat ethanol, and incubated 1 hour at $20^{\circ} \mathrm{C}$ to fix. Fixed cells were then washed gently and denatured in $2 \mathrm{M} \mathrm{HCl}$ for 20 minutes at room temperature. Following washing and 2 minute's incubation with $0.1 \mathrm{M}$ Tris to neutralize the acid, cells were re-suspended in FACS buffer (0.5\% fetal calf serum in PBS) and stained with Phycoerythrin (PE)-conjugated anti-BrdU according to the manufacturer's protocol for flow cytometry analysis on a FACScan instrument.

\section{Apoptosis and trypan blue staining}

Apoptosis was measured using a kit (BD Biosciences) containing propidium iodide $(\mathrm{PI})$ and FITC-conjugated annexin $\mathrm{V}$. Cells were stained according to the manufacturer's protocol and the level of apoptosis determined by flow cytometry. Quadrants are: early apoptosis (annexin $\mathrm{V}^{+} / \mathrm{PI}$, lower right) late apoptosis (annexin $\mathrm{V}^{+} / \mathrm{PI}+$, upper right) and non-apoptotic cell death (annexin $\mathrm{V} / \mathrm{PI}^{+}$, upper left). Treatments for the stress panel were: no treatment (control); DMSO as a control for celecoxib; $20 \mathrm{mM}$ celecoxib, brand name Celebrex ${ }^{\mathrm{TM}}$ (dissolved in DMSO) [31]; $0.2 \mathrm{mM} \mathrm{H}_{2} \mathrm{O}_{2}$ [32]; or $1 \mathrm{mg} / \mathrm{mL} \mathrm{G418}$ (Pfizer, New York, NY, USA).

\section{Real-time PCR arrays}

Transcriptional analysis using Cancer PathwayFinder $\mathrm{RT}^{2}$ profiler arrays (SuperArray, Frederick, MD, USA) was performed according to the manufacturer's protocol. Briefly, total RNA was isolated using an RNeasy extraction kit (Qiagen, Valencia, CA, USA); $1 \mu \mathrm{g}$ of RNA was reverse transcribed with the cDNA synthesis kit (SuperArray) and cDNA was subjected to real-time PCR using SYBR green to detect product. Arrays were performed independently at least twice for each cell line; all PCR products were checked on agarose gels. Values were obtained for the threshold cycle $\left(\mathrm{C}_{\mathrm{t}}\right)$ for each gene and normalized using the average of four housekeeping genes on the same array (HPRT1, RPL13A, GAPD, ACTB). $\mathrm{C}_{\mathrm{t}}$ values for housekeeping genes and a dilution series of $A C T B$ were monitored for consistency between arrays. Change $\left(\Delta \mathrm{C}_{\mathrm{t}}\right)$ between MUC1 siRNA and control siRNA was found by:

$\Delta \mathrm{C}_{\mathrm{t}}=\mathrm{C}_{\mathrm{t}(\mathrm{MUC1} \text { siRNA })}-\mathrm{C}_{\mathrm{t}(\text { control siRNA })}$

and fold change by:

Fold change $=2^{(-\Delta \mathrm{Ct})}$

Values are given as fold change; only genes showing two-fold or greater change were considered. Both luciferase and scrambled siRNA controls were used in BT-20; only genes showing consistent alteration with both controls were included in the results reported here. The scrambled siRNA could be not used in MDA-MB-468 as these cells decrease MUC1 expression in response to this construct.

\section{Results siRNA transfection decreases MUC1 expression in breast cancer cell lines}

Two human breast cancer cell lines, MDA-MB-468 and BT-20, were transiently transfected with a pool of four siRNA oligonucleotides directed against the MUC1 mRNA (468.siMUC1 and BT.siMUC1), or a control oligonucleotide directed against luciferase (468.siluc and BT.siLuc). Both cell lines express high levels of MUC1, making them promising targets for this analysis. Western blots (Figure 1a) show successful knockdown of both the extracellular domain and cytoplasmic tail 
Figure 1

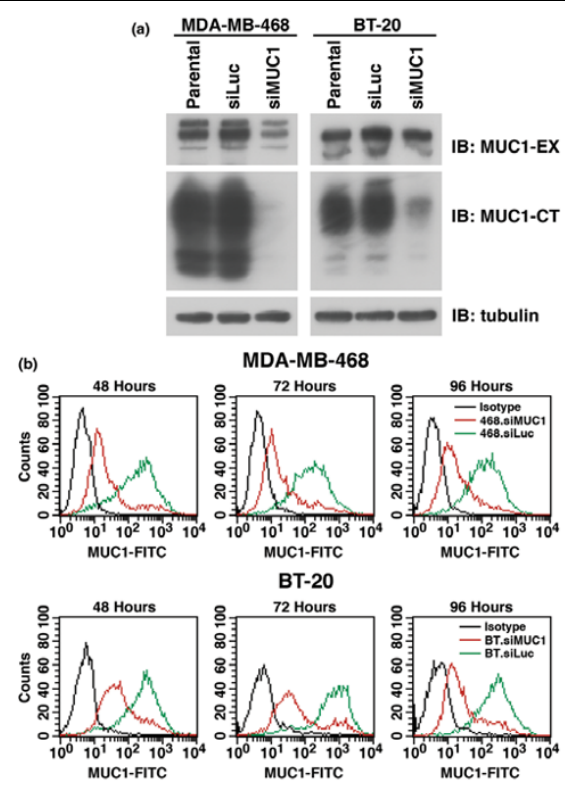

Small interfering RNA (siRNA) decreases MUC1 levels in breast cancer cells. (a) Western blots (IB) of the MUC1 cytoplasmic tail (MUC1-CT) and extracellular domain (MUC1-EX) in untreated (parental), or luciferase siRNA treated (siLuc) or MUC1 siRNA (siMUC1) treated MDAMB-468 and BT-20 cells. Lysates were analyzed 48 hours post-transfection; tubulin is shown as a loading control. (b) Flow cytometry analysis of the MUC1 extracellular domain in unpermeabilized, siRNAtreated MDA-MB-468 and BT-20 lines. Cells were analyzed at 24 hour intervals beginning 48 hours post-transfection. The black line represents isotype control, the green luciferase siRNA (siLuc), and the red MUC1 siRNA (siMUC1).

fragments of MUC1; luciferase siRNA does not substantially change the level of MUC1 compared to parental cells. 468.siMUC1 show a substantial decrease in the amount of MUC1-CT, while BT.siMUC1 show slightly less knockdown of MUC1-CT. Both MDA-MB-468 and BT-20 display a less dramatic decrease of MUC1 extracellular domain compared to MUC1-CT (Figure 1a); this likely represents protein synthesized prior to transfection, and may reflect differences in the turnover rates of the two subunits.

Analysis of the MUC1 extracellular domain by flow cytometry confirms that both cell lines substantially decrease MUC1 expression after siRNA (Figure 1b). By flow cytometry, 468.siMUC1 averaged 75\% knockdown of MUC1 compared to 468.siLuc; and BT.siMUC1 averaged 50\% knockdown relative to BT.siluc. These effects could be titrated with the concentration of siRNA, were seen as early as 24 hours posttransfection (data not shown) and lasted to at least $96 \mathrm{~h}$ posttransfection (Figure 1b). All experiments were conducted within 48 to 96 hours after siRNA transfection. Similar results were obtained using two independent oligonucleotides designed in our lab (data not shown), designated '882' and '956' for the initial codon recognized by each.
Figure 2

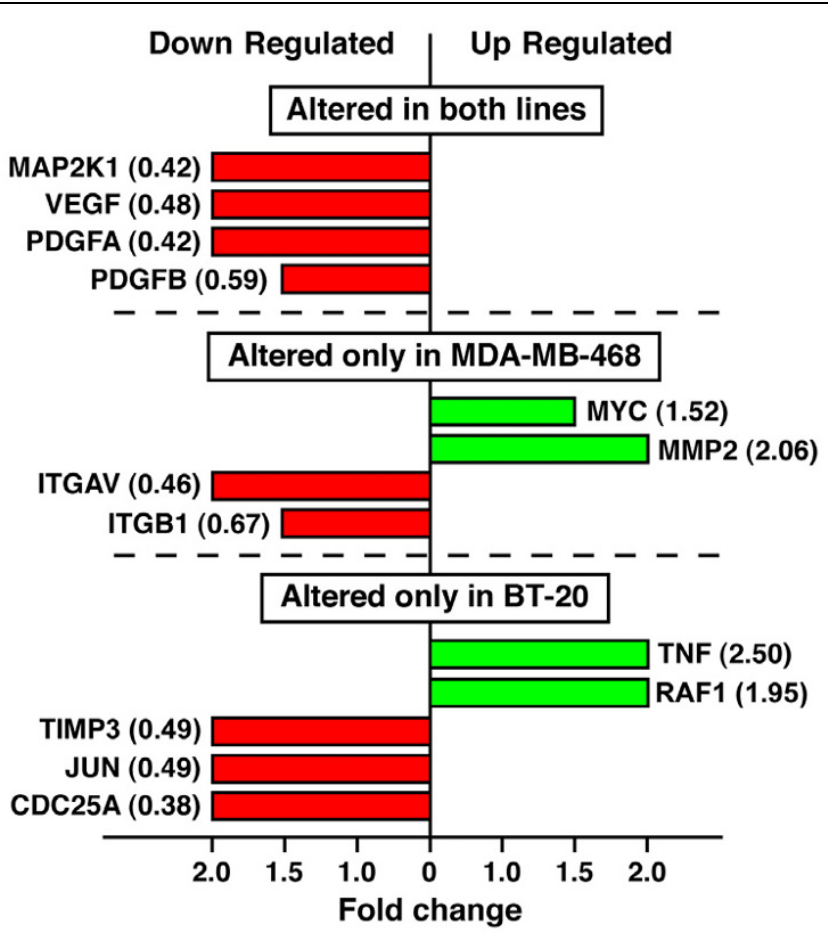

Transcription of genes involved in cancer is altered in response to MUC1 small interfering RNA (siRNA). Genes whose transcription was altered by at least two-fold in MUC1 siRNA-treated cells compared to control siRNA are shown. Green bars represent genes whose transcription was up-regulated; red bars are genes whose transcription was down-regulated after MUC1 siRNA. The average fold change is shown in parentheses after the gene name; for genes altered in both MDA-MB-468 and BT-20, these values reflect the average from both cell lines.

\section{Transcriptional changes are seen after MUC1 siRNA}

Recent work indicates that MUC1 may affect transcription both directly via interaction with transcription factors and indirectly (for example, through modulating signaling). To study the effects of MUC1 knockdown in breast cancer cell lines, real-time PCR arrays were used to analyze transcription of 84 genes implicated in cancer. Only genes with greater than twofold change were considered. Three genes (MAP2K1, VEGF, $P D G F A$ ) were altered two-fold or more after MUC1 siRNA in both MDA-MB-468 and BT-20 cells (Figure 2); two genes (ITGAV, MMP2) changed only in 468.siMUC1; and five genes ( TIMP3, RAF1, JUN, TNF, CDC25A) only in BT.siMUC1. This list represents all genes affected greater than two-fold after MUC1 siRNA, rather than a select group. Three genes whose transcription was changed by less than two-fold are shown, two of which (PDGFB and ITGB1) are listed because they relate closely to genes altered by two-fold (PDGFA and ITGAV). The third, MYC, is included because western blots confirmed a substantial change at the protein level (Figure 3a) that may reflect both transcriptional and post-transcriptional regulation. 
Figure 3

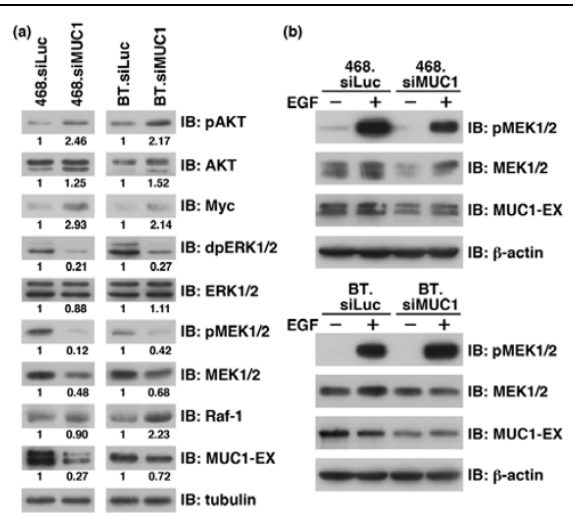

Western blot analysis confirms changes in protein levels after MUC1 small interfering RNA (siRNA). (a) Whole cell lysates were analyzed for the following proteins: MUC1 extracellular subunit (MUC1-EX), c-Myc, total and active MAPK and ERK kinase (MEK) 1/2 (MEK1/2, pMEK1/2), total and active extracellular signal regulated kinase (ERK) $1 / 2$ (ERK1/2, dpERK1/2), total and active AKT (AKT, pAKT), and tubulin as a loading control. Densitometry is shown for each blot, based on three independent measurements per band, normalized to tubulin; results are shown relative to luciferase siRNA (siLuc) for each cell line. Optimal exposures for comparing luciferase siRNA and MUC1 siRNA-treated cells from the same parental line are shown. (b) Lysates from epidermal growth factor (EGF)-stimulated MDA-MB-468 cells were blotted for MEK1/2, pMEK1/2, MUC1-EX, and actin (for loading).

Interestingly, transcription of MAP2K1 was decreased in both cell lines after MUC1 siRNA. This gene encodes MEK1, one of the primary regulators of the ERK1/2 MAPK pathway [33], a network that has been linked several times to MUC1 [12,3436]. We examined MEK1 and MEK2 levels by western blot to confirm decreased protein in MUC1 siRNA-treated cells (Figure $3 \mathrm{a}$ ), and found that not only were total MEK1/2 levels lower in 468.siMUC1 and BT.siMUC1 compared to controls ( 0.48 and 0.68 relative to siluc, respectively), but so were the basal amounts of active (phosphorylated) MEK1/2 (pMEK1/2; 0.12 and 0.42 relative to siluc, respectively). Both 468.siMUC1 and BT.siMUC1 also showed reduced activation of ERK $1 / 2$ (dpERK $1 / 2 ; 0.21$ and 0.27 relative to siLuc, respectively), as would be expected with diminished signaling through MEK1/2; total ERK1/2 levels remain unchanged.

As both lines have high levels of EGFR and thus activate the MEK-ERK cascade intensely when stimulated with EGF [37], siRNA-transfected cells were treated with EGF. Notably, MUC1 siRNA impairs this important oncogenic pathway in MDA-MB-468 cells, as 468 .siMUC1 display less pMEK $1 / 2$ in response to EGF than do 468.siluc (Figure 3b). Interestingly, EGF treatment of BT-20 cells results in slightly higher pMEK1/ 2 levels in BT.siMUC1 compared to BT.siLuc. Though this result seems paradoxical in light of decreased MAP2K1 transcription in BT.siMUC1, it likely results from differential functions of Raf isoforms in combination with the increased RAF1 transcription (Figure 2) and protein level (Figure 3a) in these cells. Specifically, B-Raf is thought to be the main activator of
Figure 4

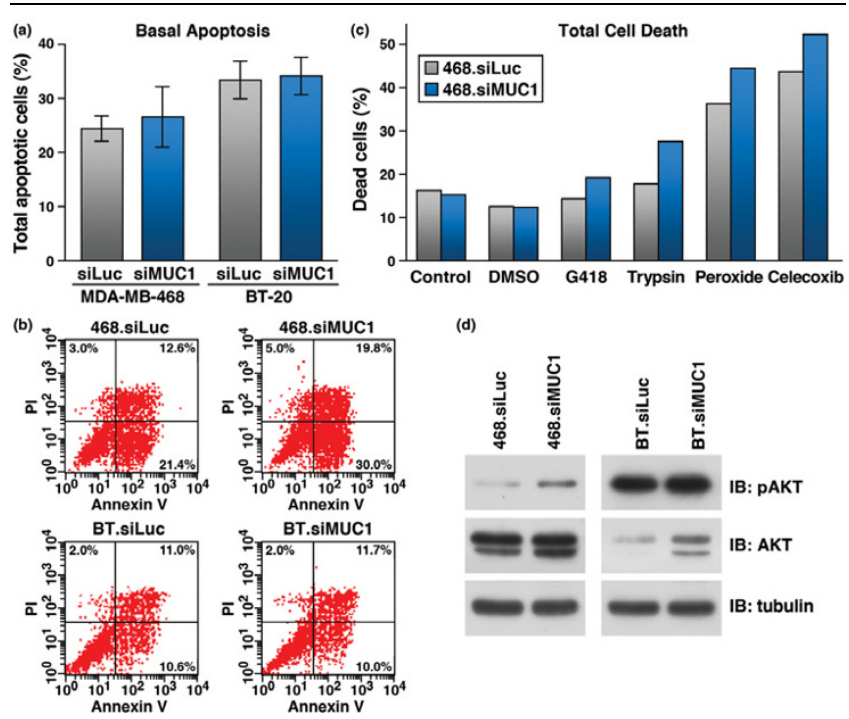

MUC1 small interfering RNA (siRNA) affects cell death in response to stress in MDA-MB-468, but not BT-20 cells. (a) Basal apoptosis is shown as the combined total of early (propidium iodide (PI)-/annexin $\left.\mathrm{V}^{+}\right)$and late $\left(\mathrm{PI}+\right.$ /annexin $\left.\mathrm{V}^{+}\right)$apoptotic populations. Results reflect the averages of three independent experiments. (b) Apoptosis after trypsinization 24 hours post-transfection. Numbers in the quadrants reflect the percentage of cells in each. Representative flow cytometry results are shown for MDA-MB-468 and BT-20 cells transfected with siRNA. (c) Total cell death (sum of non-apoptotic cell death $(\mathrm{PI}+/$ annexin $\mathrm{V}$-), and early and late apoptosis) is shown for MDA-MB-468 cells in response to a panel of cellular stresses (G418, trypsin, peroxide, celecoxib) and controls (control, DMSO). Representative results from three independent experiments are shown. (d) Western blots (IB) for total and active AKT (AKT, pAKT) and tubulin loading control, performed on whole cell lysates from siRNA transfected cells. The same exposure is shown for both cell lines to show the relative levels of protein between lines, rather than optimal exposure times for comparison within a single cell line (shown in Figure 3a).

MEK under normal conditions; Raf- 1 activates MEK in response to stimulus [38]. Thus, it appears that basal pMEK1/ 2 levels are not greatly affected by Raf- 1 overexpression in BT.siMUC1 cells, likely because MEK is regulated primarily by B-Raf under normal growth conditions. In contrast, when the cells are stimulated (EGF), increased Raf-1 levels in BT.siMUC1 leads to heightened pMEK1/2 (Figure 3b).

\section{MUC1 siRNA increases apoptosis in MDA-MB-468 but not BT-20}

We next examined whether MUC1 knockdown and its associated transcriptional alterations would affect overall cellular events. As several of the genes shown in Figure 2 are important in regulating proliferation and survival, and because of the recently described role of MUC1 in modulating apoptosis in response to cellular stresses $[20,21,24]$, we first analyzed whether MUC1 siRNA would alter apoptosis in these lines. Although there was no change in basal apoptosis in either line (Figure 4a), we observed that the cell lines responded differently when trypsinized for re-plating 24 hours after transfection 
Figure 5

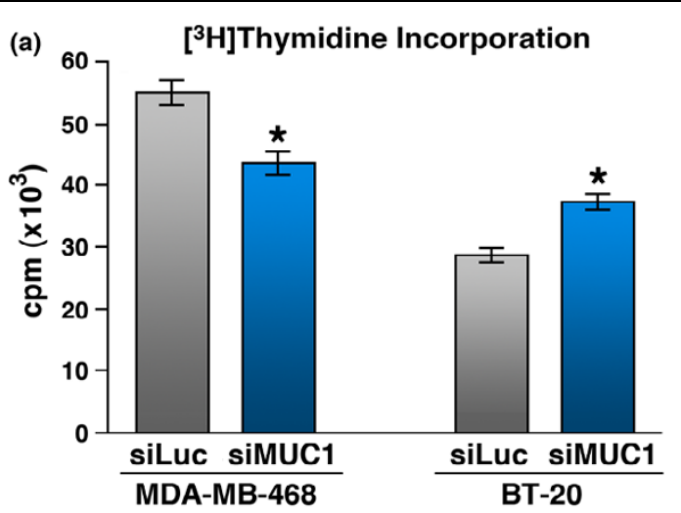

(b)
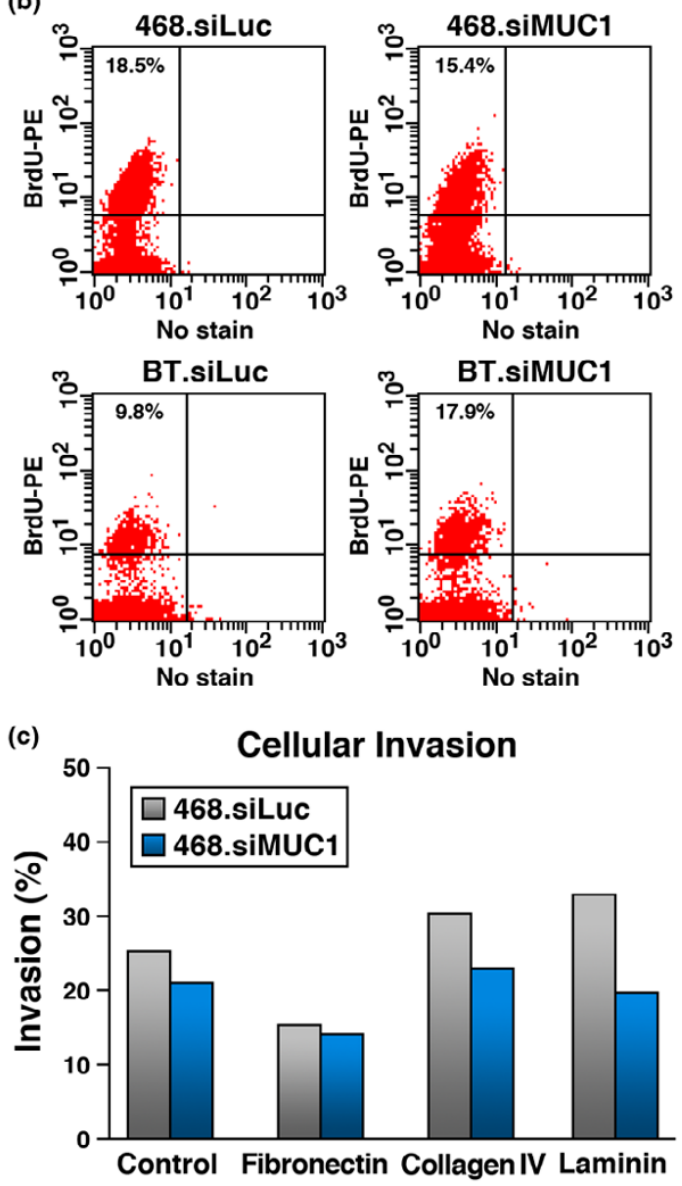

Proliferation is altered by MUC1 small interfering RNA (siRNA) in both cell lines. (a) $\left.{ }^{3} \mathrm{H}\right]$ Thymidine incorporation is shown by the level of radioactivity (cpm) emitted from cells after 24 hours incubation with radionucleotide. Results reflect the average of three independent experiments. ${ }^{*} p<0.006$ compared to luciferase siRNA (siLuc) treated cells of the same line (that is, 468.siluc or BT.siLuc). (b) Flow cytometric analysis of bromodeoxyuridine (BrdU) incorporation. siRNA-transfected cells were incubated with BrdU for 1.5 hours, and stained with a Phycoerythrin PE-conjugated antibody against BrdU. A representative experiment is shown. (c) Invasion of MDA-MB-468 cells in transwells plated with a panel of extracellular matrix proteins or no matrix (control). Representative results are shown as percent invasion, calculated as: number of invaded cells/total number of cells $\times 100$.
(Figure 4b). Interestingly, 468.siMUC1 cells show greater apoptosis after trypsinization than do 468.siluc (49.8\% versus $34.0 \%$, respectively), while BT-20 cells from both siRNA treatments display similar levels of apoptosis (around 22\%).

To examine whether this phenomenon is specific to trypsin treatment or part of a general stress response involving MUC1, we subjected cells to a panel of stresses and measured cell death. In agreement with the patterns seen with trypsinization, BT.siLuc and BT.siMUC1 respond similarly to all treatments (data not shown), while 468.siMUC1 die more readily than 468.siLuc in response to trypsin, G418, hydrogen peroxide, or celecoxib, a chemotherapeutic that targets the cyclooxygenase-2 (COX-2) pathway (Figure 4c); these data were confirmed with two independent siRNA constructs (data not shown).

Like the MAPK pathway, AKT signaling has been linked to MUC1 in cancer. Although transcription of $A K T$ was not altered in MUC1 siRNA-treated cells, the results of our apoptosis studies prompted us to investigate levels of AKT further. As expected, the total AKT protein level is not greatly changed after MUC1 siRNA in either cell line, though the active form (pAKT) is increased in both 468.siMUC1 and BT.siMUC1 compared to controls (Figure $3 \mathrm{a}$ ). This result disagrees with MUC1 activation of the AKT pathway in rat 3Y1 cells [18], and may reflect regulation more appropriate to breast cancer cells; this is supported by activation of AKT in response to MUC1 siRNA in other lines [21]. In addition, there is a striking difference in the relative amounts of AKT and PAKT in the two cell lines (Figure 4d). When lysates from both lines are exposed to film for the same length of time (overexposure masks the differences between BT.siLuc and BT.siMUC1 that are apparent in Figure $3 \mathrm{a}$ ), it is clear that PAKT levels are much higher in BT20 than in MDA-MB-468, despite lower total AKT expression. This difference in AKT activation between MDA-MB-468 and BT-20 likely contributes to the disparity in their sensitivity to the increased apoptosis expected with loss of MUC1.

\section{MUC1 siRNA alters proliferation and invasion}

As MUC1 is involved in apoptosis, we next analyzed its effects on proliferation. BrdU and $\left[{ }^{3} \mathrm{H}\right]$ thymidine incorporation were used to analyze proliferation after MUC1 siRNA. 468.siMUC1 cells show a significant decrease in $\left[{ }^{3} \mathrm{H}\right]$ thymidine incorporation compared to 468.siLuc, while intriguingly, BT.siMUC1 cells show a significant increase in proliferation (Figure $5 \mathrm{a}$ ). Growth curves mirror these results, as do experiments with the two independent MUC1 siRNA oligonucleotides (data not shown). Note that these assays require trypsinizing cells 24 hours post-transfection; therefore, the results in the MDA-MB468 line could stem from the changes in apoptosis described in the previous section, rather than a true effect on proliferation. To control for this, we incubated non-trypsinized, siRNAtransfected cells at similar confluence with BrdU to measure incorporation. The 'clumped' profile of cells (contrast to Figure 
Figure 6

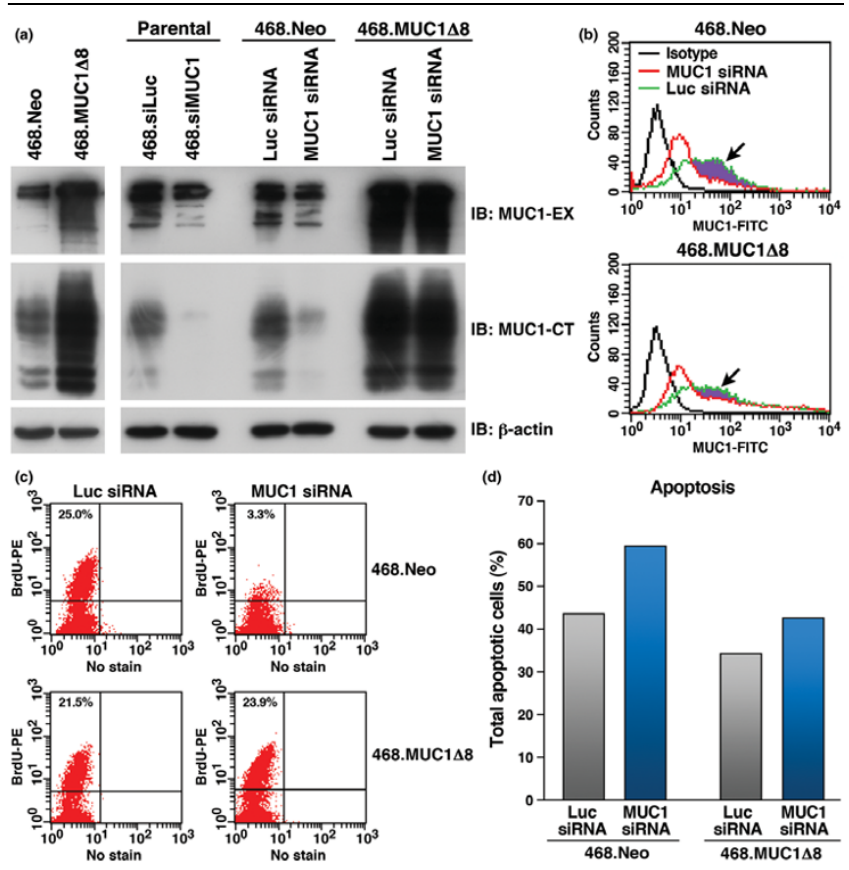

The MUC1 interfering RNA (siRNA) phenotype is rescued by stable transfection with MUC1. (a) MDA-MB-468 cells transfected with a fulllength, siRNA-resistant MUC1 construct (468.MUC1 $\triangle 8$ ) or empty vector (468.Neo); lysates were blotted for MUC1 extracellular (MUC1-EX) and cytoplasmic (MUC1-CT) domains, with actin as a loading control. The left panel shows untransfected cells; the right shows siRNA treatment of the stable transfectants and parental cells. (b) siRNA-transfected $468 . \mathrm{Neo}$ and $468 . \mathrm{MUC} 1 \Delta 8$ cells were stained for expression of MUC1-EX 48 hours post-transfection and analyzed by flow cytometry. The black line represents isotype control, the green luciferase (Luc) siRNA, and the red MUC1 siRNA. Arrows point out the shaded regions that reflect the level of knockdown in each cell line. (c) Flow cytometric analysis of bromodeoxyuridine (BrdU) incorporation after 1.5 hours incubation with siRNA transfected cells. The number shown in each upper left quadrant reflects the percentage of BrdU-positive cells. A representative experiment is shown. (d) Total apoptosis is shown as the sum of early (propidium iodide $(\mathrm{PI})$-/annexin $\mathrm{V}^{+}$) and late $\left(\mathrm{PI}^{+} /\right.$ annexin $\mathrm{V}^{+}$) apoptotic populations for $468 . \mathrm{Neo}$ and $468 . \mathrm{MUC} 1 \Delta 8$ cells trypsinized 24 hours post-transfection. A representative example is shown.

$4 b$ ) is likely a result of the acid denaturation (recommended by the antibody manufacturer), as it occurs uniformly in these experiments. BrdU incorporation (Figure 5b) confirms that the $\left[{ }^{3} \mathrm{H}\right]$ thymidine results are not solely due to alterations in apoptosis, as 468.siMUC1 cells incorporate less BrdU than 468.siLuc; once again, BT.siMUC1 cells show increased proliferation over BT.siLuc.

Given the role of MUC1 in adhesion, we examined whether MUC1 siRNA affects cellular invasion. In transwell assays, BT20 cells invaded poorly, regardless of the siRNA used (data not shown). However, MDA-MB-468 cells invade more readily, and were analyzed on a panel of three different extracellular matrix proteins. Interestingly, 468.siMUC1 cells display somewhat decreased invasion on collagen IV, laminin, and fibronec- tin matrices, and on a no-matrix control (Figure 5c), which is in agreement with the trend towards decreased metastasis observed in Muc $1 \% \times$ MMTV-PyV MT mice [8].

\section{Transfection of MUC1 rescues the 468.siMUC1 phenotype}

To determine if the above effects are specific to MUC1, we created stable transfectants of the MDA-MB-468 line using empty vector (468.Neo) or a full-length MUC1 construct (468.MUC1 $\triangle 8$ ) that is resistant to one of the independent MUC1-directed oligonucleotides ('882'). These cells were maintained in G418-containing medium to retain transgene selection. As expected, 468.MUC1 $\Delta 8$ cells show higher levels of both the MUC1 extracellular domain and the MUC1-CT than do 468.Neo (Figure 6a). Note that 468.Neo have MUC1 expression comparable to parental MDA-MB-468; the exposures in Figure $6 \mathrm{a}$ are lighter than those in Figure $1 \mathrm{a}$, in order to clearly show the relative levels of MUC1 in the stable transfectants. After MUC1 siRNA, 468.MUC1 $\triangle 8$ lose some MUC1 (likely endogenous protein, which is not siRNA-resistant) but retain high-level expression, while 468.Neo show a decrease in MUC1 levels similar to parental 468.siMUC1 cells (Figures $6 a, b)$. The difference in the amount of MUC1 knockdown between $468 . \mathrm{Neo}$ and $468 . \mathrm{MUC} 1 \Delta 8$ is highlighted by the purple shading in Figure $6 \mathrm{~b}$.

BrdU incorporation (Figure 6c) indicates that 468.Neo show decreased nucleotide incorporation after MUC1 siRNA compared to control (3.3\% versus $25.0 \%$, respectively); this is not seen in 468.MUC1 $\triangle 8$ cells, which show similar levels of BrdU incorporation regardless of the siRNA used $(21.5 \%$ for luciferase, $23.9 \%$ for MUC1). 468 .Neo cells display a more dramatic decrease in BrdU incorporation after MUC1 siRNA than what is seen in parental 468 .siMUC1 cells, which may reflect the additional stress of being maintained in G418-containing medium. Similarly, analysis of apoptosis in trypsinized cells indicates that the increased apoptosis seen in parental 468.siMUC1 cells is also present in the 468. Neo line after MUC1 siRNA (Figure 6d; $43.6 \%$ in control versus $59.6 \%$ in MUC1 siRNA). However, in $468 . M U C 1 \Delta 8$ cells, the level of apoptosis after luciferase siRNA (34.1\%) is lower than that in 468.Neo cells; MUC1 siRNA increases the amount of apoptosis slightly $(42.8 \%)$, restoring it to a level similar to that seen in luciferase siRNA-treated 468.Neo cells. Together, these studies suggest that the above-described results are specific to MUC1, as stable transfection of an siRNA-resistant MUC1 rescues the phenotype seen in 468. siMUC1 cells.

\section{Discussion}

This report describes both the transcriptional alterations seen after transfection with MUC1 siRNA in human breast cancer cells and the effects on events such as apoptosis and proliferation. The two cell lines used (MDA-MB-468 and BT-20) were chosen for high expression of MUC1 and a substantial (50\% to $75 \%$ ), consistent decrease in MUC1 expression after 
siRNA. Both lines have epithelial morphology, form tumors slowly in nude mice [37], have mutant p53 [39,40], express EGFR [37], and lack estrogen receptor $\alpha$ [41]. One striking difference between these lines, however, is their response to MUC1 siRNA. MDA-MB-468 cells behave as expected for loss of an oncogene: MUC1 siRNA correlates with increased apoptosis in response to stress, decreased proliferation, and reduced invasion. In contrast, BT.siMUC1 cells proliferate more rapidly than BT.siLuc cells with little effect on apoptosis.

Much of the phenotype of these cells can be understood in light of protein levels and transcriptional activity after MUC1 siRNA. As mentioned, both MDA-MB-468 and BT-20 display increased pAKT after MUC1 siRNA, but the ratio of active to total AKT is considerably higher in the BT-20 line, which may help these cells resist the increased apoptosis expected with loss of MUC1. Myc levels are also higher in both cell lines after MUC1 siRNA, although the ability of Myc to promote proliferation and apoptosis in different cellular contexts [42] complicates the interpretation of this finding.

Both cell lines show reduced transcription of VEGF, PDGFA, $P D G F B$, and MAP2K1 (MEK1) after MUC1 siRNA. The genes encoding vascular endothelial growth factor (VEGF) and the $A$ and $B$ chains of platelet-derived growth factor (PDGF-A and PDGF-B) are interesting as these proteins have been heavily implicated in angiogenesis, suggesting a novel function for MUC1 in regulating this process. Vascular endothelial growth factor expression in cancer is linked to tumor growth and metastasis $[43,44]$; platelet-derived growth factor is also angiogenic, but has an additional role in stimulating desmoplasia [45]. Reduced transcription of these genes after MUC1 siRNA suggests that MUC1 may foster angiogenesis and stromal proliferation, although this must be confirmed in a more appropriate model system.

Decreased MAP2K1 (MEK1) transcription after MUC1 siRNA provides a novel mechanism by which MUC1 can affect the ERK1/2 MAPK pathway. MUC1 has often been linked to the Ras-Raf-MEK-ERK cascade [12,34-36,46], and at least two mechanisms by which MUC1 can alter MAPK signaling have been described: MUC1 interaction with and phosphorylation by the EGFR family [12,13], and MUC1 binding to the Grb2/ Sos complex that activates Ras [46]. Reduction of MEK1 levels after MUC1 siRNA agrees with the role of MUC1 in strengthening MAPK signaling, and indicates that MUC1 can regulate both the transcription and activity of members of this pathway.

Two additional MAPK pathway members are altered specifically in BT.siMUC1, with no corresponding change in 468.siMUC1 cells. These genes are RAF1 and JUN which are increased and decreased, respectively, after MUC1 siRNA. Raf- 1 and c-Jun both function outside of the ERK1/2 MAPK pathway, which may explain the seeming paradox of increased
RAF1 transcription with simultaneous decreases in MAP2K1 and JUN. Specifically, Raf-1 can inhibit ASK1 (apoptosis signal-regulated kinase 1) upstream of p38 and JNK (Jun N-terminal kinase) [38]. ASK1 phosphorylates JNK in response to stress, resulting in activation of c-Jun and stimulation of apoptosis [47], indicating that the coordinate up-regulation of RAF1 and down-regulation of JUN may provide a potent antiapoptotic effect in BT.siMUC1.

Regulation of life and death is also a hallmark of the CDC25A and TNF gene products. CDC25A is a phosphatase that stimulates cell cycle progression [48], thus the effects of its decrease in BT.siMUC1 are unclear in light of the increased proliferation of these cells. However, the CDC25 proteins (A, $B$, and $C$ ) were recently shown to have greater functional overlap than was previously thought [49], suggesting that the other two isoforms may compensate for reduced CDC25A levels. $T N F$ encodes tumor necrosis factor (TNF) $\alpha$, known for its potent, cell type-specific control of life and death. In tumor cells, TNF $\alpha$ expression can promote proliferation and inhibit apoptosis [50], suggesting that increased $T N F$ transcription in BT.siMUC1 could contribute to the increased proliferation seen in these cells.

Interestingly, the increase in $T N F$ is accompanied by decreased transcription of TIMP3, encoding tissue inhibitor of metalloproteinases (TIMP)3. The TIMP family disrupts the function of matrix metalloproteinases (MMPs), generally resulting in decreased invasion [51]. TIMP3 is unique in that it can also inhibit TNF $\alpha$ converting enzyme (TACE), which activates TNF $\alpha$ by cleaving it from the cell surface [50]. Reduced expression of TIMP3 would, therefore, foster signaling through TNF $\alpha$ by releasing inhibition of TNF $\alpha$ converting enzyme. In agreement with this, TIMP3 can promote apoptosis [52]; thus, its down-regulation in BT.siMUC1 provides another mechanism by which these cells are able to resist the increased apoptosis expected with loss of MUC1.

Another TIMP target responds to MUC1 siRNA, as 468.siMUC1 cells show significantly increased expression of MMP2 (encoding MMP-2/gelatinase A), the product of which degrades type IV collagen [52]. In breast cancer, the ratio of active to latent MMP-2 increases with tumor progression; MMP-2 may facilitate both angiogenesis and metastasis [52]. Its increase after loss of MUC1 is, therefore, unexpected, but at least two factors may clarify this result. First, though MMP2 levels are increased in mouse mammary tumors, its expression is confined to the stroma [53], suggesting that increased MMP2 transcription after loss of the epithelium-specific MUC1 might reflect a shift towards a more mesenchymal phenotype. Second, MMP-2 levels are increased by overexpression of erbB2 [52]; previous studies have shown that erbB2 and Muc1 expression are mutually exclusive in mammary tumors [27], implying that MMP2 might be part of a transcriptional profile linked to low MUC1 levels. 
It is intriguing that, despite increased MMP2 transcription, invasion is decreased in 468.siMUC1 cells, even on collagen IV. This may reflect insufficient activation of MMP-2, as the precursor protein must be cleaved for enzymatic function [52]. Alternatively, the slowed invasion of these cells may relate to impaired adhesion resulting from decreased transcription of ITGAV and ITGB1 ( $\alpha_{v}$ and $\beta_{1}$ integrins, respectively). Integrin signaling is tied to life-or-death decisions in epithelial cells, and integrin expression is vital for processes from wound healing to metastasis [54]. Integrin $\alpha_{v} \beta_{3}$ is implicated in facilitating metastasis of breast cancer cells to bone [55]; decreased transcription of ITGAV after MUC1 siRNA may, therefore, suggest that MUC1 is involved in this lethal process as well.

The MUC1 oncogene has been linked to apoptosis $[18,20,26]$, proliferation [17], and transcription [21,23-25] in cancer. However, the two cell lines chosen for our study display very different responses to MUC1 siRNA, indicating that regulation of MUC1 in breast cancer is likely quite complex and cautioning against over-generalization of results from individual cell lines. Previous reports suggest that, though most studies outline a clearly oncogenic role for MUC1 in breast cancer, the exact details may vary depending on factors such as cell type and signaling context. For example, MUC1 stimulates Fas-mediated apoptosis in $\mathrm{CHO}$ cells [26], quite unlike the inhibition of apoptosis seen in other cell lines. Similarly, though MUC1 drives mammary oncogenesis in its own right [9] and facilitates tumorigenesis driven by other oncogenes $[7,8]$, Muc1 is selectively down-regulated in c-neu-induced mouse mammary tumors [27], indicating that the context of oncogenic signaling is vital to understanding the function of MUC1.

Thus, it is important to consider the relative levels of knockdown of MUC1 in the two cell lines: BT-20 cells reduce MUC1 expression after siRNA less strongly than do MDA-MB-468 (50\% versus $75 \%$ knockdown, respectively). As MUC1 serves as a scaffold [11], overexpression of MUC1 relative to its associated signaling proteins might create a dilution effect, sequestering signal transducers away from each other; this would be relieved by MUC1 siRNA. Thus, enough MUC1 may be retained in BT.siMUC1 cells for its oncogenic effects, while signaling complex formation would be enhanced by lowering the amount of MUC1 relative to other signaling proteins.

\section{Conclusion}

The contrast between the MDA-MB-468 and BT-20 lines in response to MUC1 siRNA serves as a reminder that simplified models such as cell lines fail to encompass the complexity of intact biological systems. This report describes transcriptional alterations seen after MUC1 knockdown: decreased transcription of MAP2K1, VEGF, PDGFA, ITGAV, TIMP3, CDC25A, and $J U N$, and increased transcription of MMP2, TNF, and $R A F 1$. The alterations in MAP2K1, RAF1, and JUN represent a novel means by which MUC1 can affect ERK1/2 signaling: transcriptional regulation of MAPK pathway members. Oncogenic events are also altered in both cell lines after MUC1 siRNA. These results strengthen the growing ties linking MUC1 and transcriptional regulation, and suggest that the role of MUC1 in breast cancer may be more complex than a direct correlation between MUC1 level and oncogenic function.

\section{Competing interests}

The authors declare that they have no competing interests.

\section{Authors' contributions}

CLH performed all studies and composed the manuscript. SJG participated in the design and coordination of the studies and contributed strongly to the revision of the manuscript. Both authors have read and approved the manuscript.

\section{Acknowledgements}

We thank Pinku Mukherjee for statistics, Eric Thompson for cloning the MUC1 silent mutations, Kandavel Shanmugam for cloning into the pLNCX.1 vector, Teresa Tinder for technical assistance, Gargi Basu for experimental expertise, Irene Beauvais for administrative assistance, and Marv Ruona for graphics support. This work was supported by the Department of Defense Breast Cancer pre-doctoral fellowship W81XWH-04-1-0300 (CLH) and the National Institutes of Health R01 CA64389 (SJG).

\section{References}

1. Gendler SJ: MUC1, the renaissance molecule. J Mammary Gland Biol Neoplasia 2001, 6:339-353.

2. Baldus SE, Engelmann K, Hanisch F-G: MUC1 and the MUCs: a family of human mucins with impact in cancer biology. Crit Rev Clin Lab Sci 2004, 41:189-231.

3. Regimbald LH, Pilarski LM, Longenecker BM, Reddish MA, Zimmermann G, Hugh JC: The breast mucin MUC1 as a novel adhesion ligand for endothelial intercellular adhesion molecule 1 in breast cancer. Cancer Res 1996, 56:4244-4249.

4. Lillehoj EP, Kim BT, Kim KC: Identification of Pseudomonas aeruginosa flagellin as an adhesin for Muc1 mucin. Am J Physiol Lung Cell Mol Physiol 2002, 282:L751-756.

5. Carraway KL 3rd, Ramsauer VP, Carothers Carraway CA: Glycoprotein contributions to mammary gland and mammary tumor structure and function: roles of adherens junctions, ErbBs and membrane MUCs. J Cell Biochem 2005, 96:914-926.

6. Braun DP, Crist KA, Shaheen F, Staren ED, Andrews S, Parker J: Aromatase inhibitors increase the sensitivity of human tumor cells to monocyte-mediated, antibody-dependent cellular cytotoxicity. Am J Surg 2005, 190:570-571.

7. Schroeder JA Adriance MC, Thompson MC Camenisch TD, Gendler SJ: MUC1 alters beta-catenin-dependent tumor formation and promotes cellular invasion. Oncogene 2003, 22:1324-1332.

8. Spicer AP, Rowse GJ, Lidner TK, Gendler SJ: Delayed mammary tumor progression in Muc-1 null mice. J Biol Chem 1995, 270:30093-30101.

9. Schroeder JA, Masri AA, Adriance MC, Tessier JC, Kotlarczyk KL, Thompson MC, Gendler SJ: MUC1 overexpression results in mammary gland tumorigenesis and prolonged alveolar differentiation. Oncogene 2004, 23:5739-5747.

10. Li $Y$, Kuwahara H, Ren J, Wen G, Kufe D: The c-Src tyrosine kinase regulates signaling of the human DF3/MUC1 carcinoma-associated antigen with GSK3 beta and beta-catenin. $J$ Biol Chem 2001, 276:6061-6064.

11. Al Masri A, Gendler SJ: Muc1 affects c-Src signaling in PyV MTinduced mammary tumorigenesis. Oncogene 2005, 24:5799-5808.

12. Schroeder JA, Thompson MC, Gardner MM, Gendler SJ: Transgenic MUC1 interacts with epidermal growth factor receptor and correlates with mitogen-activated protein kinase activa- 
tion in the mouse mammary gland. J Biol Chem 2001, 276:13057-13064.

13. Li Y, Ren J, Yu W, Li Q, Kuwahara H, Yin L, Carraway KL 3rd, Kufe $\mathrm{D}$ : The epidermal growth factor receptor regulates interaction of the human DF3/MUC1 carcinoma antigen with c-Src and beta-catenin. J Biol Chem 2001, 276:35239-35242.

14. Meerzaman D, Shapiro PS, Kim KC: Involvement of the MAP kinase ERK2 in MUC1 mucin signaling. Am J Physiol Lung Cell Mol Physiol 2001, 281:L86-91.

15. Pandey $P$, Kharbanda S, Kufe D: Association of the DF3/MUC1 breast cancer antigen with Grb2 and the Sos/Ras exchange protein. Cancer Res 1995, 55:4000-4003.

16. Steelman LS, Pohnert SC, Shelton JG, Franklin RA, Bertrand FE McCubrey JA: JAK/STAT, Raf/MERK/ERK, PI3K/Akt and BCR$A B L$ in cell cycle progression and leukemogenesis. Leukemia 2004, 18:189-218.

17. Li X, Wang L, Nunes DP, Troxler RF, Offner GD: Suppression of MUC1 synthesis downregulates expression of the epidermal growth factor receptor. Cancer Biol Ther 2005, 4:968-973.

18. Raina D, Kharbanda S, Kufe D: The MUC1 oncoprotein activates the anti-apoptotic phosphoinositide 3-kinase/Akt and Bcl-xL pathways in rat 3Y1 fibroblasts. J Biol Chem 2004, 279:20607-20612.

19. Ren J, Agata N, Chen D, Li Y, Yu WH, Huang L, Raina D, Chen W, Kharbanda S, Kufe D: Human MUC1 carcinoma-associated protein confers resistance to genotoxic anticancer agents. Cancer Cell 2004, 5:163-175.

20. Yin L, Li Y, Ren J, Kuwahara H, Kufe D: Human MUC1 carcinoma antigen regulates intracellular oxidant levels and the apoptotic response to oxidative stress. J Biol Chem 2003, 278:35458-35464

21. Yin L, Huang L, Kufe D: MUC1 oncoprotein activates the FOXO3a transcription factor in a survival response to oxidative stress. J Biol Chem 2004, 279:45721-45727.

22. Wen Y, Caffrey TC, Wheelock MJ, Johnson KR, Hollingsworth MA: Nuclear association of the cytoplasmic tail of MUC1 and betacatenin. J Biol Chem 2003, 278:38029-38039.

23. Huang L, Ren J, Chen D, Li Y, Kharbanda S, Kufe D: MUC1 cytoplasmic domain coactivates Wnt target gene transcription and confers transformation. Cancer Biol Ther 2003, 2:702-706.

24. Wei X, Xu H, Kufe D: Human MUC1 oncoprotein regulates p53responsive gene transcription in the genotoxic stress response. Cancer Cell 2005, 7:167-178.

25. Wei $\mathrm{X}, \mathrm{Xu} \mathrm{H}$, Kufe D: MUC1 oncoprotein stabilizes and activates estrogen receptor alpha. Mol Cell 2006, 21:295-305.

26. Chaturvedi R, Srivastava RK, Hisatsune A, Shankar S, Lillehoj EP, Kim KC: Augmentation of Fas ligand-induced apoptosis by MUC1 mucin. Int J Oncol 2005, 26:1169-1176.

27. Adriance MC, Gendler SJ: Downregulation of Muc1 in MMTV-cNeu tumors. Oncogene 2004, 23:697-705.

28. Liu H, Radisky DC, Nelson CM, Zhang H, Fata JE, Roth RA, Bissell MJ: Mechanism of Akt1 inhibition of breast cancer cell invasion reveals a protumorigenic role for TSC2. Proc Natl Acad Sci 2006, 103:4134-4139.

29. Mukherjee P, Tinder TL, Basu GD, Gendler SJ: MUC1 (CD227) interacts with Ick tyrosine kinase in Jurkat lymphoma cells and normal T cells. J Leukoc Biol 2005, 77:90-99.

30. Image] [http://rsb.info.nih.gov/ij/index.html]

31. Basu GD, Pathangey LB, Tinder TL, Gendler SJ, Mukherjee P: Mechanisms underlying the growth inhibitory effects of the cyclo-oxygenase-2 inhibitor celecoxib in human breast cancer cells. Breast Cancer Res 2005, 7:R422-435.

32. Troyano A, Sancho P, Fernandez C, de Blas E, Bernardi P, Aller P: The selection between apoptosis and necrosis is differentially regulated in hydrogen peroxide-treated and glutathionedepleted human promonocytic cells. Cell Death Differ 2003, 10:889-898.

33. Johnson GL, Lapadat R: Mitogen-activated protein kinase pathways mediated by ERK, JNK, and p38 protein kinases. Science 2002, 298:1911-1912.

34. Meerzaman D, Shapiro PS, Kim KC: Involvement of the MAP kinase ERK2 in MUC1 mucin signaling. Am J Physiol Lung Cell Mol Physio/ 2001, $281:$ L86-91.

35. Wang H, Lillehoj EP, Kim KC: MUC1 tyrosine phosphorylation activates the extracellular signal-regulated kinase. Biochem Biophys Res Commun 2004, 321:448-454.
36. Lillehoj EP, Kim H, Chun EY, Kim KC: Pseudomonas aeruginosa stimulates phosphorylation of the airway epithelial membrane glycoprotein Muc1 and activates MAP kinase. Am J Physiol Lung Cell Mol Physiol 2004, 287:L809-815.

37. Arteaga CL, Hurd SD, Dugger TC, Winnier AR, Robertson JB: Epidermal growth factor receptors in human breast carcinoma cells: a potential selective target for transforming growth factor alpha-Pseudomonas exotoxin $\mathbf{4 0}$ fusion protein. Cancer Res 1994, 54:4703-4709.

38. Baccarini M: Second nature: Biological functions of the Raf-1 "kinase". FEBS Lett 2005, 579:3271-3277.

39. Elstner E, Linker-Israeli M, Said J, Umiel T, de Vos S, Shintaku IP Heber D, Binderup L, Uskokovic M, Koeffler HP: 20-epi-vitamin D3 analogues: a novel class of potent inhibitors of proliferation and inducers of differentiation of human breast cancer cell lines. Cancer Res 1995, 55:2822-2830.

40. Do TN, Rosal RV, Drew L, Raffo AJ, Michl J, Pincus MR, Friedman FK, Petrylak DP, Cassai N, Szmulewicz J, et al.: Preferential induction of necrosis in human breast cancer cells by a p53 peptide derived from the MDM2 binding site. Oncogene 2003, 22:1431-1444.

41. Lindner DJ, Borden EC: Synergistic antitumor effects of a combination of interferon and tamoxifen on estrogen receptorpositive and receptor-negative human tumor cell lines in vivo and in vitro. J Interferon Cytokine Res 1997, 17:681-693.

42. Dang CV, O'Donnell KA, Juopperi T: The great MYC escape in tumorigenesis. Cancer Cell 2005, 8:177-178.

43. Robinson CJ, Stringer SE: The splice variants of vascular endothelial growth factor (VEGF) and their receptors. J Cell Sci 2001, 114:853-865.

44. Augustin HG: Translating angiogenesis research into the clinic: the challenges ahead. Br J Radiology 2003, 76:S3-S10.

45. Shao ZM, Nguyen M, Barsky SH: Human breast carcinoma desmoplasia is PDGF initiated. Oncogene 2000, 19:4337-4345.

46. Pandey P, Kharbanda S, Kufe D: Association of the DF3/MUC1 breast cancer antigen with Grb2 and the Sos/Ras exchange protein. Cancer Res 1995, 55:4000-4003.

47. Takeda K, Matsuzawa A, Nishitoh H, Ichijo H: Roles of MAPKKK ASK1 in stress-induced cell death. Cell Struct Funct 2003, 28:23-29.

48. Boutros R, Dozier C, Ducommun B: The when and wheres of CDC25 phosphatases. Curr Opin Cell Biol 2006, 18:185-191.

49. Lindqvist $A$, Kallstrom $H$, Lundgren $A$, Barsoum $E$, Rosenthal CK: Cdc25B cooperates with Cdc25A to induce mitosis but has a unique role in activating cyclin $\mathrm{B} 1-\mathrm{Cdk} 1$ at the centrosome. $J$ Cell Biol 2005, 171:35-45

50. Mocellin S, Rossi CR, Pilati P, Nitti D: Tumor necrosis factor, cancer and anticancer therapy. Cytokine Growth Factor Rev 2005, 16:35-53.

51. Brew K, Dinakarpandian D, Nagase H: Tissue inhibitors of metalloproteinases: evolution, structure and function. Biochim Biophys Acta 2000, 1477:267-283.

52. Duffy MJ, Maguire TM, Hill A, McDermott E, O'Higgins N: Metalloproteinases: role in breast carcinogenesis, invasion, and metastasis. Breast Cancer Res 2000, 2:252-257.

53. Blavier L, Lazaryev A, Dorey F, Shackleford GM, DeClerck YA: Matrix metalloproteinases play an active role in Wnt1-induced mammary tumorigenesis. Cancer Res 2006, 66:2691-2699.

54. van der Flier A, Sonnenberg A: Function and interactions of integrins. Cell Tissue Res 2001, 305:285-298.

55. Sloan EK, Anderson RL: Genes involved in breast cancer metastasis to bone. Cell Mol Life Sci 2002, 59:1491-1502. 